\title{
Relapse of Tourette Syndrome with Clozapine in a Patient of Paranoid Schizophrenia
}

\author{
Sandeep Grover*, Prabhakar Pokhrel and Natasha Kate \\ Department of Psychiatry, Postgraduate Institute of Medical Education \& Research, Chandigarh 160012, India
}

\begin{abstract}
Tourette syndrome (TS) is characterized by multiple motor and vocal tics and is commonly associated obsessiveCompulsive Disorder (OCD). Comorbidity between TS and schizophrenia has been less commonly described. In this case report a case of schizophrenia with comorbid TS is described in which symptoms of TS relapsed when the patient was started on clozapine. Exacerbation of TS with clozapine has been reported in few case reports in the literature. Our case highlights the fact that clozapine should be used with caution in patients with schizophrenia who have tic disorder.
\end{abstract}

\section{Introduction}

Tourette syndrome (TS) is characterized by multiple motor and vocal tics occurring for at least one year. It is commonly associated with attention deficit hyperactivity disorder (ADHD) and obsessivecompulsive disorder (OCD) [1,2]. Other comorbid psychiatric disorders reported to be seen in patients with Tourette syndrome include autistic disorder, Asperger's syndrome and bipolar disorder [3-5]. However, there is limited data on comorbidity of Tourette syndrome with schizophrenia [6,7]. Further, only few case reports suggest worsening of tics with clozapine [8-11].

In this case report we present a case who had Tourette syndrome prior to adolescence, which resolved on its own. Later the patient developed schizophrenia and while receiving clozapine, had a relapse of symptoms of Tourette syndrome.

\section{Case Description}

Mr X, 21 yr old, single, High school passed, presented to the inpatient unit with diagnoses of paranoid schizophrenia and akathisia while receiving olanzapine and aripiprazole. Detailed evaluation of the history revealed that since early childhood, he had motor tics in the form of jerky involuntary movements of the shoulder and hand along with vocal tics, which peaked prior to teen age and then reduced significantly. Initially motor tics were not associated with any dysfunction, but as the frequency and intensity of the tics increased, these were associated with marked social dysfunction. Additionally he had obsessive compulsive behaviour in the form of fear of contamination and repeated washing rituals since early childhood, which led to mild dysfunction. These symptoms gradually receded by the age of 12-13 years along with disappearance of motor tics.

Around the age of 15 years, without any precipitating factor, he developed symptoms characterized by suspiciousness, violent behaviour, delusion of reference, persecution, grandiosity, social withdrawal, anhedonia, apathy, amotivation, associated with academic decline and marked psychosocial dysfunction. He was seen by a psychiatrist after few months of onset of symptoms and was started on Tab amisulpiride which was continued for about 2 months, with which he showed no improvement in symptoms. Following this he was treated with adequate trials of aripiprazole, risperidone, olanzapine and a combination of aripiprazole and olanzapine. Further the history revealed that whenever he received olanzapine, either alone or in combination, he developed severe akathisia.
There was no family history of mental illness or tics. On examination, patient was restless and kept on pacing around. He had delusion of reference, persecution, grandiosity and thought insertion.

A diagnosis of paranoid schizophrenia (as per DSM-IV) and drug induced akathisia was made. His positive and negative symptom scale (PANSS) score was 75 and his Global Clinical Assessment of akathisia on Barnes akathisia rating scale (BARS) was 4 . Tab aripiprazole and olanzapine were stopped. In view of the treatment history and side effects, clozapine was considered. Prior to starting clozapine his heamogram, liver function test, renal function test, serum electrolytes, lipid profile, fasting blood glucose level, electrocardiogram and electroencephalogram did not reveal any abnormality. Clozapine was started at the dose of $25 \mathrm{mg} /$ day and gradually increased to $250 \mathrm{mg} /$ day over the period of 4 weeks. Additionally he was treated with Tab clonazepam (up to $3 \mathrm{mg} /$ day) and Tab propranolol $40 \mathrm{mg} /$ day. With clozapine, patient showed significant improvement in his psychotic symptoms and akathisia also reduced significantly. By 6 weeks of clozapine therapy, his PANSS score came down to 41 and the Global Clinical Assessment of Akathisia on Barnes akathisia rating scale (BARS) was rated as 1 (i.e., questionable akathisia). All along his haematological monitoring did not reveal any abnormality. He was discharged from the inpatient unit at this time.

In follow up he reported occasional positive symptoms, following which the dose of clozapine was increased to $275 \mathrm{mg} /$ day. Within few days of increase in the dose of clozapine to $275 \mathrm{mg}$ /day, patient developed motor tics involving the shoulder and elbow along with vocal tics in the form of uttering some religious words. Over next few days he also developed obsessive compulsive symptoms, which were associated with marked distress. Following this clozapine was reduced to $250 \mathrm{mg} /$

*Corresponding author: Dr. Sandeep Grover, Assistant Professor, Department of Psychiatry, Postgraduate Institute of Medical Education \& Research, Chandigarh 160012, India, Tel: 0091-172-2756807; Fax: +91-172-2744401, 2745078; E-mail: drsandeepg2002@yahoo.com

Received February 14, 2012; Accepted February 20, 2012; Published February 25, 2012

Citation: Grover S, Pokhrel P, Kate N (2012) Relapse of Tourette Syndrome with Clozapine in a Patient of Paranoid Schizophrenia. J Clinic Case Reports 2:e112. doi:10.4172/2165-7920.1000e112

Copyright: $\odot 2012$ Grover S, et al. This is an open-access article distributed under the terms of the Creative Commons Attribution License, which permits unrestricted use, distribution, and reproduction in any medium, provided the original author and source are credited. 
day and a repeat electroencephalogram was done, which did not reveal any abnormality. Neurologist opinion was sought, who considered the possibility of a tic disorder (Tourette's syndrome). Following this he was started on Tab sertraline $50 \mathrm{mg} /$ day, with which tics and obsessive compulsive symptoms reduced significantly over the period of 5-6 weeks. He has been now maintaining well with Tab clozapine $250 \mathrm{mg} /$ day and Tab sertraline $50 \mathrm{mg} /$ day and is functioning well.

\section{Discussion}

TS is associated with many comorbid psychiatric disorders. Among the various comorbidities, schizophrenia is less commonly reported. In a study involving 399 TS patients, schizophrenia was seen in $2.5 \%$ of cases [12]. Other studies have reported prevalence of schizophrenia like symptom in about $11-12 \%[7,13]$ and schizotypal or schizoid personality disorders in $13-15 \%$ of patients with TS $[14,15]$.

In general it is reported that most of the patients with TS have reduction in tics and obsessive compulsive features by adolescence [16], as was in the index case. However, an interesting finding noted in the index case was relapse of symptoms of TS with clozapine. Similar relationship between worsening of tics with clozapine has been described in the form of case reports in the literature [8-11]. In another small study, authors reported exacerbation of TS in 4 out of 7 patients with starting of clozapine in low doses [17]. Although underlying mechanism is not known, some of the authors suggest that worsening of tics with clozapine may be related to an imbalance between the dopamine and serotonin systems [10].

In their review of literature, Marsalek [11] reported that there are 15 reported cases of tardive TS secondary to use of neuroleptic for more than 2 years in patients with schizophrenia. Another 2 cases of tardive TS were reported later [18]. Further, previous reports of tardive TS have reported the same while the patients were receiving an antipsychotic (8 cases) or after stoppage of neuroleptics (9 cases). In the index case, symptoms of TS had a relapse after being treated with clozapine for few months but shortly after increase in the dose from $250 \mathrm{mg} /$ day to $275 \mathrm{mg} /$ day, and symptoms reduced with reduction in dose of clozapine and addition of sertraline. As the index case had received multiple antipsychotics for duration of more than 5 years, possibility of tardive TS can also be considered in this case. However, none of the previous report had noted existent of the symptoms of TS prior to antipsychotic treatment as was seen in the index case. Hence, we considered the possibility of relapse of TS rather than the possibility of tardive TS.

Our case highlights the fact that TS patients can go on to develop schizophrenia later on and patients of schizophrenia, especially early onset schizophrenia may have history of comorbid TS. In view of the same when ever a patient pr@esents with either of the diagnosis, proper evaluation should include evaluation for the other disorder. Additionally our case suggests that clozapine should be used with caution in patients with Tourette syndrome, as it can lead to worsening of the overall symptomatology. Further, in view the literature, it is important to remember than long term use of antipsychotic can lead to development of tardive TS.

\section{References}

1. Freeman RD (2007) Tic disorders and ADHD: answers from a world-wide clinical dataset on Tourette syndrome. Eur Child Adolesc Psychiatry 16: 15-23.
2. Freeman RD, Fast DK, Burd L, Kerbeshian J, Robertson MM, et al. (2000) An international perspective on Tourette syndrome: selected findings from 3,500 individuals in 22 countries. Dev Med Child Neurol 42: 436-447.

3. Burd L, Kerbeshian J (1987) A North Dakota prevalence study of schizophrenia presenting in childhood. J Am Acad Child Adolesc Psychiatry 26: 347-350.

4. Kerbeshian J, Burd L, Klug MG (1995) Comorbid Tourette's disorder and bipolar disorder: an etiologic perspective. Am J Psychiatry 152: 1646-1651.

5. Kerbeshian J, Burd L (1996) Case study: comorbidity among Tourette's syndrome, autistic disorder and bipolar disorder. J Am Acad Child Adolesc Psychiatry 35: 681-685.

6. Kerbeshian J, Burd L (1987) Are schizophreniform symptoms present in attenuated form in children with Tourette disorder and other developmental disorders. Can J Psychiatry 32: 123-135.

7. Kerbeshian J, Burd L (1986) Asperger's syndrome and Tourette syndrome: the case of the pinball wizard. Br J Psychiatry 148: 731-736.

8. Bastiampillai T, Dhillon R, Mohindra R (2008) Exacerbation of tics secondary to clozapine therapy. Aust N Z J Psychiatry 42: 1068-1070.

9. Begum M (2005) Clozapine-induced stuttering, facial tics and myoclonic seizures: a case report. Aust N Z J Psychiatry 39: 202.

10. Poyurovsky M, Bergman Y, Shoshani D, Schneidman M, Weizman A (1998) Emergence of obsessive-compulsive symptoms and tics during clozapine withdrawal. Clin Neuropharmacol 21: 97-100.

11. Marsalek M (2000) Tardive drug induced extrapyramidal syndromes Pharmacopsychiatry 33: 14-33.

12. Kerbeshian J, Peng CZ, Burd L (2009) Tourette syndrome and comorbid earlyonset schizophrenia. J Psychosom Res 67: 515-523.

13. Comings DE, Comings BG (1987) A controlled study of Tourette syndrome. IV. Obsessions, compulsions and schizoid behaviors. Am J Hum Genet 41: 782-803.

14. Robertson MM, Banerjee S, Hiley PJ, Tannock C (1997) Personality disorder and psychopathology in Tourette's syndrome: a controlled study. $\mathrm{Br} \mathrm{J}$ Psychiatry 171: 283-286.

15. Cavanna AE, Robertson MM, Critchley HD (2007) Schizotypal personality traits in Gilles de la Tourette syndrome. Acta Neurol Scand 116: 385-391.

16. Bloch MH, Peterson BS, Scahill L, Otka J, Katsovich L, et al. (2006) Adulthood outcome of tic and obsessive-compulsive symptom severity in children with Tourette syndrome. Arch Pediatr Adolesc Med 160: 65-69.

17. Caine ED, Polinsky RJ, Kartzinel R, Ebert MH (1979) The trial use of clozapine for abnormal involuntary movement disorders. Am J Psychiatry 136: 317-320.

18. Yamauchi K, Ohmori T (2006) Two cases of tardive Tourette syndrome. Seishin Shinkeigaku Zasshi 108: 459-465. 